\title{
Interpretation of the cosmic-ray air shower signal in Askaryan radio detectors
}

\author{
Krijn D. de Vries ${ }^{1, \star}$, Stijn Buitink ${ }^{2}$, Nick van Eijndhoven ${ }^{1}$, Thomas Meures ${ }^{3}$, Aongus \\ O'Murchadha $^{3}$, and Olaf Scholten ${ }^{1,4}$ \\ ${ }^{1}$ Vrije Universiteit Brussel, Dienst ELEM, IIHE, Pleinlaan 2, 1050 Brussels, Belgium \\ ${ }^{2}$ Vrije Universiteit Brussel, Astrophysical Institute, Pleinlaan 2, 1050 Brussels, Belgium \\ ${ }^{3}$ Dept. of Physics and Wisconsin IceCube Particle Astrophysics Center, University of Wisconsin, Madison, \\ WI 53706,USA \\ ${ }^{4}$ University of Groningen, KVI-Center for Advanced Radiation Technology, 9747 AA Groningen, The Nether- \\ lands
}

\begin{abstract}
We discuss the radio emission from a cosmic-ray air shower propagating in air before it hits an air-ice boundary after which it completes its propagation inside the ice. The in-air emission, the in-ice emission, as well as the transition radiation from the shower crossing the boundary is considered. We discuss the interpretation of the radio signal observed by an in-ice observer.
\end{abstract}

\section{Introduction}

In [1], we presented a calculation for the radio emission from a cosmic-ray air shower hitting an ice surface. It is shown that this signal should be observable by the currently existing Askaryan radio detectors [2-4]. The in-air emission, the in-ice emission, as well as the coherent transition radiation from the boundary were considered. In this article we focus on the interpretation of the observed signal and discuss the properties of the coherent transition radiation in view of the sudden appearance and sudden death signals. The sudden appearance signal is for example seen during beamtest experiments, for which the beam is hidden to the observer (within the observing frequency band) while inside the accelerator, after which it 'suddenly' becomes visible while exiting the accelerator. An example of the sudden death signal is the signal originating from a beam-dump process.

The importance of the cosmic-ray air shower signal in Askaryan radio detectors, if identified correctly, lies in its possibility to calibrate the currently existing Askaryan radio detectors. Furthermore, if detected, this signal immediately shows the on-site feasibility of the Askaryan radio detection technique. If misidentified, however, this signal might pose a background in the search for the signal from a high-energy neutrino-induced particle cascade in ice.

\section{Macroscopic modeling}

The calculations in [1] are based on a macroscopic calculation [5- -7], starting from the LiénardWiechert potentials from classical electrodynamics. The electric field is directly obtained from the

\footnotetext{
^e-mail: krijndevries@gmail.com
} 
charge and current distributions in the cascade front. The fields have to be evaluated at the negative retarded emission time, $t_{r}$, which links to the observer time, $t$, through the optical path length $L$ from the emission point to the observer,

$$
c\left(t-t_{r}\right)=L
$$

The optical path length inside a medium consisting out of $m$ layers with different index of refraction $n_{i}$, can be defined by

$$
L=\sum_{i=1}^{m} n_{i} d_{i}
$$

Here the distance $d_{i}$, traversed by the emission in layer $i$, is obtained by using a ray-tracing procedure based on Snell's law.

The in-air, as well as the in-ice emission originates from the time-variation of the total number of particles in the cascade, $d N\left(t_{r}\right) / d t_{r}$, in combination with relativistic boosting effects. In this article, however, we will focus on the coherent transition radiation and its link to the sudden appearance and sudden death signal. In [1], the coherent transition radiation signal was given by,

$$
\vec{E}_{t r}(t, \vec{x})=\lim _{\epsilon \rightarrow 0} \int \mathrm{d}^{2} \vec{r} \frac{e d N_{e}\left(t_{r}\right) w(\vec{r}, h)}{4 \pi \epsilon_{0} c} \times\left.\left(\frac{1}{|\mathcal{D}|_{t_{r}-\epsilon}^{2}}-\frac{1}{|\mathcal{D}|_{t_{r}+\epsilon}^{2}}\right) \hat{p}\right|_{h=c\left(t_{r}-t_{b}\right)} .
$$

Following the same procedure the sudden appearance signal is given by,

$$
\vec{E}_{s a}(t, \vec{x})=-\left.\lim _{\epsilon \rightarrow 0} \int \mathrm{d}^{2} \vec{r} \frac{e d N_{e}\left(t_{r}\right) w(\vec{r}, h)}{4 \pi \epsilon_{0} c|\mathcal{D}|_{t_{r}+\epsilon}^{2}} \hat{p}\right|_{h=c\left(t_{r}-t_{b}\right)} .
$$

Here $d$ denotes the impact distance from the observer to the shower axis, where $w(\vec{r}, h)$ denotes the particle distribution in the cascade front at lateral position $\vec{r}$ and a distance $h$ behind the cascade front. The retarded distance $\mathcal{D}$ is defined by,

$$
\mathcal{D}=L \frac{d t}{d t_{r}}
$$

The obtained fields agree well with the microscopic calculations presented in [8-[11], and references therein.

The coherent transition radiation obtained within this formalism can be understood as the emission just above the air-ice boundary at $t_{r}-\epsilon$, and the emission just below the boundary at $t_{r}+\epsilon$. Linking Eqs. 3 and 4, the coherent transition radiation can be interpreted as the superposition of the sudden appearance signal and the sudden death signal. As will be shown in the following section, under the proper circumstances these signals will indeed be observed separately in the case of coherent transition radiation.

It should be noted that these solutions are limiting solutions. The derivation of the vector potential from Maxwell's equations has to be considered with great care, since this is done under the assumption of a continuous medium, where in our situation we consider a hard boundary. This is reflected by the fact that $t_{r}(t)$ is discontinuous at the boundary, and hence the retarded distance is ill defined at this point.

\section{Results and interpretation}

How are these results reflected in our simulation? This is shown in Fig. (1), where we show the signal observed for a perpendicular incoming air shower which penetrates the ice at an elevation of $3 \mathrm{~km}$, 

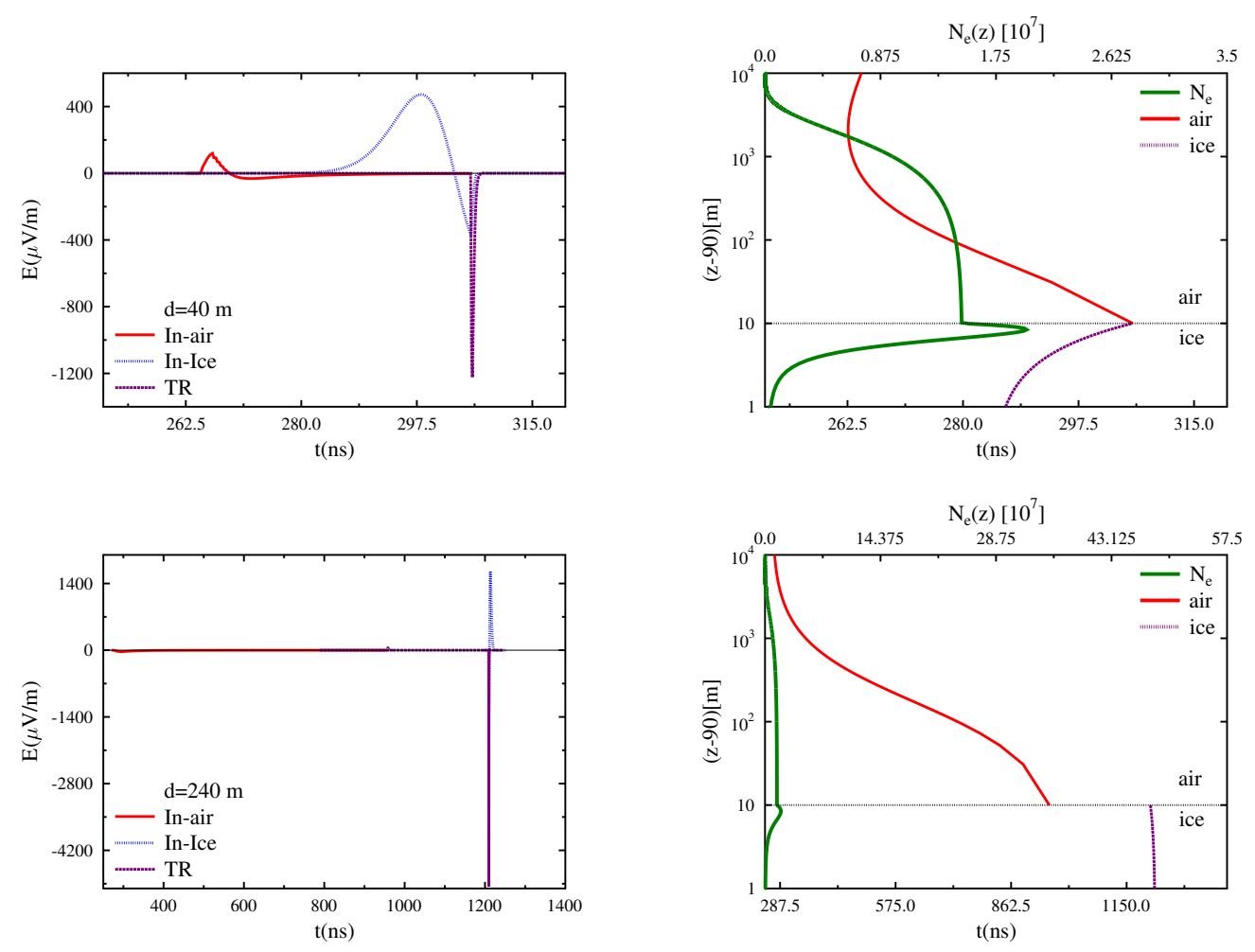

Figure 1. The electric field at different observer distances equal to, a) $d=40 \mathrm{~m}$, b) $d=240 \mathrm{~m}$. The figures on the right show the emission height, plotted as function of the observer time. The full red line gives the emission in air, the dotted purple line gives the transition radiation, and the dashed blue line gives the in-ice emission. For the figures on the right, the total number of particles is given by the full green line. Figure and caption taken from [1].

after which the cascade completes its propagation inside the ice. The observer is positioned in the ice, $100 \mathrm{~m}$ below the air-ice boundary. The detailed particle distributions are given in [1].

The top figure shows the signal observed by an observer located inside the in-ice Cherenkov cone, but outside the in-air Cherenkov cone. The interpretation of this signal can be seen in the top-right figure, where we show the emission height, linked to the emission time by $z=-c t_{r}$, as function of the observer time. For the in-air emission, since the observer is positioned outside the Cherenkov cone, early emission from large heights is seen before late emission, where the emission from close to the boundary is observed latest. The in-ice emission, since seen from inside the Cherenkov cone, is seen differently. Late emission is seen before early emission. It also follows that the in-ice emission is observed at the same time as the in-air emission. Lastly the emission from just above and just below the boundary superimpose to give the coherent transition radiation component.

The bottom figure shows a very interesting situation. Here the point where the cascade hits the boundary is both outside the in-air, as well as outside the in-ice Cherenkov cone. Since the in-ice Cherenkov angle also denotes the critical angle, the in-air emission only enters the ice at this angle. 
Hence, the emission from just above the boundary first travels through the air, before it breaks into the ice at the critical angle. Even though this is the longer path, since the signal is not delayed by the medium for its in-air travel, it will arrive before the signal emitted from just below the boundary which traverses its full path to the observer inside the ice. It follows that in these geometries, the in-air and the in-ice emission are completely separated in time. Hence, the observer will first observe the emission from the in-air cascade, after which it appears that the cascade suddenly dies out. The dying out of the cascade is given by the sudden death signal. After some delay, from the observer point of view, the cascade suddenly appears after which the in-ice emission is seen. Hence in this situation, the emission just above the boundary is completely separated from the emission just below the boundary. Therefore, the transition radiation is not seen at a single time, but becomes separated in time. Within this framework, therefore the more fundamental description is given by the sudden appearance and sudden death signals. The observation of such a signal separated in time would confirm the interpretation of coherent transition radiation as the superposition of a the more fundamental sudden appearance and sudden death signals.

\section{Summary}

We discussed the interpretation of the coherent transition radiation signal, which originates from a cosmic-ray-induced air shower traversing between air and ice. It follows that within the given formalism, this can be interpreted as the superposition of two more fundamental signals given by the cascade sudden appearance and sudden death signals. This interpretation should be taken with care, since the derived potentials on which the calculations are based implicitly rely on the assumption of a continuous medium. This is reflected in the fact that the retarded distance is ill defined at the air-ice boundary. To solve for the fields, the limiting situation of the emission just above and just below the air-ice boundary is considered. The superposition of these two components gives rise to the coherent transition radiation signal. It is shown that in certain geometries, where the observer is positioned outside the in-ice Cherenkov cone for emission directly below the air-ice boundary, the coherent transition radiation is split in two signal separated in time. These signals, the sudden appearance signal and the sudden death signal, can therefore within the given formalism be interpreted as more fundamental.

\section{References}

[1] K.D. de Vries et al., Astropart. Phys. 74, 96-104 (2016)

[2] P.W. Gorham et al., Phys. Rev. Lett. 103, 051103 (2009)

[3] P. Allison et al., ARA Collaboration, Astropart. Phys. 35, 457-477 (2012)

[4] A. Nelles et al., ARIANNA Collaboration, these proceedings

[5] O. Scholten, K. Werner, F. Rusydi, Astropart. Phys. 29, 94-103 (2008)

[6] K.D. de Vries, A.M. van den Berg, O. Scholten, K. Werner, Phys. Rev. Lett. 107, 061101 (2011)

[7] K. Werner, K.D. de Vries, O. Scholten, Astropart. Phys. 37, 5-16 (2012)

[8] V.L. Ginzburg, V.N. Tsytovich, Transition Radiation and Transition Scattering, Adam Hilger Press, New York, 1990

[9] S. ter Veen et al., Phys. Rev. D 82, 103014 (2010)

[10] C.W. James, H. Falcke, T. Huege, M. Ludwig, Phys. Rev. E 84, 056602 (2011)

[11] P. Motloch, J. Alvarez-Muñiz, P. Privitera, E. Zas, Phys. Rev. D 93, 043010 (2016) 